\title{
MMAPDNG: A new, fast code backed by a memory-mapped database for simulating delayed $\gamma$-ray emission with MCNPX package
}

Tak Pui Lou*, Bernhard Ludewigt

Acceleration $\&$ Fusion Research Division, Lawrence Berkeley National Laboratory 1 Cyclotron Road, Berkeley, CA 94720, USA

\begin{abstract}
The simulation of the emission of beta-delayed gamma rays following nuclear fission and the calculation of time-dependent energy spectra is a computational challenge. The widely used radiation transport code MCNPX includes a delayed gamma-ray routine that is inefficient and not suitable for simulating complex problems. This paper describes the code "MMAPDNG" (MemoryMapped Delayed Neutron and Gamma), an optimized delayed gamma module written in $\mathrm{C}$, discusses usage and merits of the code, and presents results. The approach is based on storing required Fission Product Yield (FPY) data, decay data, and delayed particle data in a memory-mapped file. When compared to the original delayed gamma-ray code in MCNPX, memory utilization is reduced by two orders of magnitude and the ray sampling is sped up by three orders of magnitude. Other delayed particles such as neutrons and
\end{abstract}

\footnotetext{
*Corresponding author is a former LBNL employee. Permanent phone number: +1(510)325-3191. Permanent postal address: Travessa dos Anjos No. 37 Edifício Po Ka Yun $\mathrm{R} / \mathrm{CH} \mathrm{Bl.} \mathrm{4,} \mathrm{Macau.}$

Email addresses: TakPui.Lou@gmail.com (Tak Pui Lou), Bernhard_Ludewigt@LBL.gov (Bernhard Ludewigt)
}

Preprint submitted to Computer Physics Communications

March 14, 2015

(C) 2015. This manuscript version is made available under the Elsevier user license http://www.elsevier.com/open-access/userlicense/1.0/ 
electrons can be implemented in future versions of MMAPDNG code using its existing framework.

Keywords: delayed gamma, fission products, MCNPX, mmap

\section{Introduction}

High-energy, beta-delayed gamma-ray spectroscopy has been studied in recent years as a promising method to detect fissile isotopes and to assay nuclear materials, e.g. in spent fuel assemblies. [1 4] The distribution of short-lived fission products generated by neutron or photon induced fission is characteristic of a fissile (or fissionable) isotope. These characteristics are reflected in the emission of delayed gamma rays and can be exploited to determine the relative concentrations of the fissile isotopes from measured delayed gamma-ray spectra. For the development of practical delayed gamma techniques simulation studies are needed that use high-fidelity models to adequately describe the complexity in real life situations.

The simulation of a delayed gamma measurement involves several steps starting with the transport of source particles (e.g. from a neutron source) into an object and inducing fission. A post-processing code may be used to generate a delayed gamma-ray spectrum for a specific period that can serve as the source term for a subsequent simulation of the transport into a detector and of the detector response. With the interests in simulating beta-delayed gamma-ray emissions for a wide variety of scenarios and improving accuracy, strong modeling capabilities are needed. However, there is currently no available code that does not have serious computational limitations. 


\subsection{Delayed Gamma-ray Code in MCNPX - a loosely coupled function call approach}

MCNPX is a versatile Monte Carlo particle transport code that is widely used in the safeguards community. Delayed particle emission has been implemented since version 2.6.c, released in December 2006. [5] However, the calculation of emitted beta-delayed gammas based on discrete energy line data was fragile and researchers reported the delayed gamma code was broken in its early beta testing versions. [6]

The delayed gamma code in MCNPX has not substantially been improved in its last release version 2.7.0, one issue being that the code wastes a lot of memory and computer time. The delayed gamma code in MCNPX can be considered a "loosely coupled" function call approach because the MCNPX developers simply converted the CINDER90 Fortran program into Fortran subroutines. [7] When there is a fission event, the code makes a call to the CINDER90 subroutines and a full blown of depletion calculation is performed with one hundred time bins defined in log scale. This is similar to the loosely coupled external program approach except that the delayed gammas are emitted automatically from the exact position where fission occurs.

In this approach, the code stores three hundred time-dependent spectra for each fissioning isotope and each spectrum can contain up to tens of thousands of discrete gamma line data resulting in a large memory requirement. Normally, a modern computer should have more than enough memory to handle these gamma line data if shared memory is used to store the nuclear data in the distributed multiprocessing version (i.e. MPI version) of MCNPX. Unfortunately, the MPI version of MCNPX stores one copy of its own data per 
process. To reduce the memory requirement, the MCNPX developers chose to write their own caching code to limit the memory requirement by storing only a few selected time-dependent gamma spectra. When the spectra are not in the cache, the code re-performs the full blown depletion analysis with another CINDER90 function call. Besides the inefficient use of computing resources, this ultimately created more problems than it solved. We observed that when MCNPX is run with full discrete data (i.e. thresh $\approx 1$ of the ACT card in the input file), incorrect gamma peaks can appear and some correct peaks can disappear in the result, likely due to a bug in the caching code.

After obtaining the time-dependent delayed gamma spectrum, the code then samples the gamma energy by searching through it. Depending on the time domain of interest, the accuracy of the result can be adversely affected by the pre-defined time bin width of these spectra. Also, the MCNPX code samples the other fission product of a fission product pair in a fission event by inappropriately treating fission product yield data as fission fragment yield data. In addition, most computers would not have enough resources to run complex simulation models (e.g. a spent fuel assembly with 264 different fuel pins) with the delayed gamma code in MCNPX.

\subsection{A loosely coupled external program approach}

The problems with the delayed gamma code in MCNPX has earlier led to the development of the Discrete Gamma-ray Source DEFinition (DGSDEF) code, which is an external program loosely coupled to MCNPX. This code has been used for studying delayed gamma techniques for nuclear safeguard applications. [8 10] In this approach, CINDER90 is run as a standalone program after running MCNPX to calculate the thermal, fast and high-energy 
fission rates in discrete spatial zones. Then, the DGSDEF code post-processes the CINDER90 output and calculates the delayed gamma spectra. The calculated zone-specific gamma spectra are fed back into MCNPX for an independent delayed gamma transport simulation.

While the DGSDEF code approach is relatively easy to implement, there are disadvantages to the loosely coupled external program approach: The delayed gammas are emitted from an approximated spatial distribution rather than the exact locations of the fission events which may reduce the accuracy of the simulation results depending on how complex the modeled geometry is. In addition, output data from one step has to be converted to the correct input for the next step. Furthermore, the authors have not made the DGSDEF code available to other researchers.

\section{MMAPDNG Code}

The MMAPDNG code described in the following sections was developed to overcome the issues of the existing code in MCNPX including waste of computing resources, inefficiency, and slow execution as discussed above. Our work is based on MCNPX 2.7.0 (i.e. the last and final release of MCNPX).[11, 12

MMAPDNG takes a new approach that is radically different from the codes discussed in Section 1. Most distinctly, the fission product yield data are converted to cumulative distribution function (CDF) data while the delayed particle emission data are saved as pre-sorted and pre-calculated CDF data. When a fission event occurs, the code samples two random numbers to get two independent fission products and samples at least two other random 
numbers to obtain the delayed gamma emission time and energies. The code continues to sample more random numbers to obtain delayed gammas from the daughters' decays until a set cutoff time is reached.

\subsection{Nuclear Data Structure in File-Backed Memory}

The pre-calculated data used by MMAPDNG is obtained from a preprocessing program called dng written specifically to convert the ASCII formatted cinder dat and cindergl. dat data in MCNPX/CINDER90 into a memory-mapped file. There are several advantages of this file-backed memory approach: (a) it minimizes the initialization time as this conversion is a one-time process; (b) it eliminates unnecessary computation during the delayed gamma-ray sampling process as CDF data are needed for the simulation; and, (c) it only reserves memory for one copy of the data for as many MCNPX processes as the user requests because the file-backed memory is shared by the MCNPX running processes on a computer node.

Figure 1 shows how the data are arranged in memory. Each fissioning isotope (e.g. ${ }^{235} \mathrm{U},{ }^{238} \mathrm{U}$ or ${ }^{239} \mathrm{Pu}$ ) is stored as an array of pointers to fission products with their associated CDF data. Using the sampled random numbers, an interpolation search is performed to obtain the parent fission products. Fission product data structures of isobars (i.e. FP chain 1, FP chain 2 , etc.) are packed together in memory to minimize the cache misses. These fission product data structures contain decay data such as decay constants, branching ratios, pointers to 25-group delayed gamma emission data, pointers to discrete delayed gamma line data, pointers to continuous delayed gamma data, etc. Additional random numbers are sampled to determine the production time, delayed gamma energies, and decay branch. If a nuclide 
has more than one decay daughter product, the data structure contains additional data pointing to its daughter nuclide on another decay chain. This data structure enables all fissioning isotopes to share all these fission product decay data thus greatly reducing the memory footprint.

\subsection{Enhancements for Modeling Complex Problems}

In addition to the data structure presented in Section 2.1, a number of features are implemented to speed up the simulation. One minor but valuable feature implemented is a hybrid linear-interpolation search function to speed up the sampling of discrete gamma line data. A hybrid search algorithm is advantageous because the discrete gamma line data are pre-sorted according to the sampling probability. Many fission products have a few high 
probability gamma lines. Therefore, the linear search is often adequate and efficient. When a fission product has many gamma lines with low sampling probability, the interpolation search algorithm is usually a better choice.

Although the search algorithms implemented in MMAPDNG are very efficient and fast, merely speeding up the delayed gamma sampling process is not enough for efficient simulation of more complex problems such as the delayed gamma-ray response of a fully heterogenous model of a spent fuel assembly. Other important features for modeling complex scenarios are described in the following sections.

\subsubsection{Automatic Energy and Angular Biasing}

Two straightforward to implement features are (a) energy biasing and (b) angular biasing. Energy biasing is important for applications that are interested in the high-energy portion of the delayed gamma spectrum, which often only makes up a small fraction of the entire spectrum. When fission products emit delayed gamma energies greater than $2.7 \mathrm{MeV}$ (i.e. BIASENERGY variable in the MMAPDNG source code), biased CDF data are pre-calculated and inserted into the mmaped decay data file (i.e. \$TMPDIR/cinder.mmap) by the dng external data preprocessing program. MMAPDNG samples the gamma energy according to the biased CDF data and adjusts the particle weight to account for the energy biasing. Equations 1 and 2 show the biased probability, $p_{\gamma_{i}}$ biased, and its weight adjustment factor, $C_{\gamma_{i}}$, for the $i$-th gamma emitted by a fission product. The biased CDF data are based on a rigorously tested user-defined relationship $p_{\gamma_{i} \text { biased }} \propto E_{\gamma_{i}}^{1.5}$. This energy biasing is done automatically and the hardcoded BIASENERGY parameter in the cidDat.h header file of MMAPDNG can be used to turn it off (e.g. setting 
BIASENERGY $=100 \mathrm{MeV})$.

$$
\begin{aligned}
p_{\gamma_{i} \text { biased }} & =E_{\gamma_{i}}^{1.5} \frac{\sum_{\text {all } i} p_{\gamma_{i}}}{\sum_{\text {all }_{i} E_{\gamma_{i}}^{1.5}}} \\
C_{\gamma_{i}} & =\frac{p_{\gamma_{i}}}{p_{\gamma_{i} \text { biased }}}
\end{aligned}
$$

where $E_{\gamma_{i}}$ is the energy for the $i$-th gamma emitted by a fission product and $p_{\gamma_{i}}$ is its unbiased emission probability.

Angular biasing is necessary when the gamma detector is at a distance from the nuclear material or has a limited field of view of the nuclear material. MMAPDNG is capable of biasing the isotropic angular distribution of the emitted delayed gamma rays. Equation 3 shows the biased probability function used.

$$
\begin{aligned}
p_{\mu \text { biased }} & =\frac{1}{c} \exp [(\mu+1) a] ; \text { for }-1 \leq \mu \leq 1 \\
\text { where } a & =-\ln (1-0.99|T|) \\
\int_{-1}^{1} p_{\mu \text { biased }} d \mu & =1 \\
\therefore c a & =\exp (2 a)-1 \\
\mu_{\text {biased }} & =\ln (c a \xi+1) / a-1 ; \text { for } 0 \leq \xi<1
\end{aligned}
$$

The biased emission cosine angle, $\mu_{\text {biased }}$, is sampled with a uniform random number $\xi$ from Equation 7 during the simulation where $c$ in Equations 3, 6 and 7 is an arbitrary constant obtained by setting the integral Equation 5 equal to 1 . The particle weight of such angular biased delayed gamma is then adjusted by $0.5 / p_{\mu}$ biased. The way to activate this angular 
biasing feature for delayed gammas is by turning on exponential transform with a constant stretching parameter (i.e. $T$ in Eq. 4) very close to 0. A negative sign of the stretching parameter means the emitted delayed gamma is biased away from the stretching vector direction as defined by the VECT card while positive sign means toward the stretching direction. To prevent overflow or underflow error, Equation 4 converts $T$ into " $a$ " which is always a positive number.

Recommended stretching parameters are $\leq 0.05$ for cells with fission materials and zero for other cells. Because the stretching parameters are simultaneously used by exponential transform variance reduction technique, users should be aware of the consequence of applying exponential transform in his/her simulation. A very small stretching parameter (e.g. 0.01) usually has very little effect in terms of exponential transform but can still improve the statistical errors of delayed gammas emitting toward the biased direction.

\subsubsection{Delayed Gamma-ray Generation Time Selection}

The energy and angular biasing techniques allow the user to have some control on the statistical errors due to the physics of radioactive decay of fission products and due to the geometric arrangements of detectors. However, a lot of computer time may still be wasted if the user is interested only in a subset of the delayed gamma-rays emitted during the measurement. For example, a sample may be irradiated for one minute and counted for another minute repeatedly until enough counts are acquired. By immediately terminating the delayed gamma-ray emission during the irradiation time, a significant portion of computer time can be saved. MMAPDNG allows a user to select the delayed gamma generation time by adding new keywords in the 
surface source write (i.e. SSW) card in the input file. 11 The usage of these new keywords is discussed in Section 2.3.

\subsubsection{Particle Tag in Source Tape and Automatic Weight Control}

Particle tagging in MMAPDNG simulation result is accomplished by extending the original surface source tape code in MCNPX (i.e. a form of list mode output file) to include a particle tag in each record instead of using the particle tagging feature in a tally. The original particle tag uses the standard MCNPX notation (i.e. ZZAAA), which can be problematic for denoting nuclear isomers ${ }^{2}$ MMAPDNG adopted the AAAZZZM notation used by CINDER90 for the delayed gamma tag instead of ZZAAA for several reasons, most notably a surface source tape file is required as a workaround to obtain correct pulse-height tally results with MCNPX.

The surface source read (SSR) card was updated to accept the new surface source tape with particle tag $3^{3}$ A newly introduced keyword "dng=N" for the

\footnotetext{
${ }^{1}$ We chose to add a new keyword in the SSW card instead of using the rdum card for this user input parameter because the rdum array is generally used to provide user input parameters in MCNPX's user-supplied subroutines (e.g. source.F, srcdx.F and tallyx.F). Avoiding the use of rdum can prevent possible conflict between MMAPDNG and user-supplied subroutines.

${ }^{2} \mathrm{ZZ}$ means atomic number and AAA means mass number. The metastable states in MCNPX are denoted by $A A A+(M+3) \cdot 100$ where $M$ is the metastable state number and greater than zero. Such a notation requires a pre-defined maximum mass number for some isotopes in the code to convert this obscured AAA value into a metastable state number.

${ }^{3}$ To speed up the initialization of running MCNPX in parallel with a large surface source tape to read, the surface source read code was rewritten to mmap the surface source
} 
SSR card is mandatory for obtaining correct pulse-height (i.e. F8 or type 8) tally results for delayed particles generated internally with MCNPX. MCNPX treats all delayed gamma-rays from a single fission event as one single history and a F8 tally sums the energies deposited by all these interacted gammas from a single history to give a single pulse/score. In other words, the F8 approach assumes that a detector sees all detected gammas from one fission event as a single pulse regardless when these gammas reach the detector. This way of handling the delayed gamma-rays is wrong as the radioactive decay times involved are long enough for the gamma rays to be considered as separate scores in a detector. 4 For that reason, a F8 tally must be performed in a subsequent run from a surface source tape containing delayed gamma rays treated as separate histories. Our workaround allows the subsequent run, when using the $d n g=N$ keyword, to split all these delayed gamma rays into separate histories so that an F8 tally can work properly. For N $>1$, each record in the surface source tape can be further split into $\mathrm{N}$ histories with equally divided particle weight.

The surface source tape augmented with particle tag provides event-byevent reaction information and thus a better means to understand the simulated results. Using an external program (e.g. mapsrc and detResp), a user

\footnotetext{
tape to prevent making multiple copies over the network.

${ }^{4}$ Simultaneously emitted particles (e.g. prompt fission gammas or decay gammas from a single daughter nuclide) should be treated as correlated particles. However, most of the scenarios we concern about are deep penetration problems. Therefore, we assume that these correlated particles from one decay event do not reach a detector simultaneously. Without this assumption, we need a more sophisticated code to handle these correlated particles.
} 
can analyze the contributions of specific fission products to a spectrum. With MMAPDNG and mapsrc, a user can identify delayed gamma rays from fission products with bad statistical variances. For example, high-energy scattered gamma rays can introduce artifacts in a simulated spectrum when the incident directions of a gamma ray is close to the scattered direction produced by a next event estimator (i.e. DXTRAN sphere). Figure 2 shows the simulated spectra measured from a spent fuel assembly with $4 \%$ initial enrichment, 30 GWd/MTU burnup and 5 yrs of cooling time. By examining the augmented surface source tape with our own mapsrc C code, the three red peaks circled in green in Figure 2(a) are diagnosed to be artifacts created by three highly forward scattered high-energy photons. The surface source tape reveals that these peaks are three separate single events with a Compton scatter tag. These spikes protruding from the relatively smooth bremsstrahlung and Compton curve are typical errors found in highly forward scattered next event estimators.

In MCNPX, such an artifact from a coherent scattered photon due to next event estimators is well documented in Ref. [13] and the $\mathrm{NOCOH}$ parameter in the photon physics card (i.e. phys:p) can be used to suppress such artifact within the detailed physics treatment when DXTRAN sphere is used in a simulation. Figure 2(b) shows a spectrum without NOCOH set to 0 . The mapsrc analysis code was able to identify that the factitious portion of the peak at $\sim 3.45 \mathrm{MeV}$ in Figure 2(b) is due to a single history with a coherent scattered particle tag (i.e. 0 ). While there is $\mathrm{NOCOH}=1$ as a quick fix for such artifact from coherent scattered photons, there is no easy way to correct this artifact from high-energy Compton scattered photons other than making 

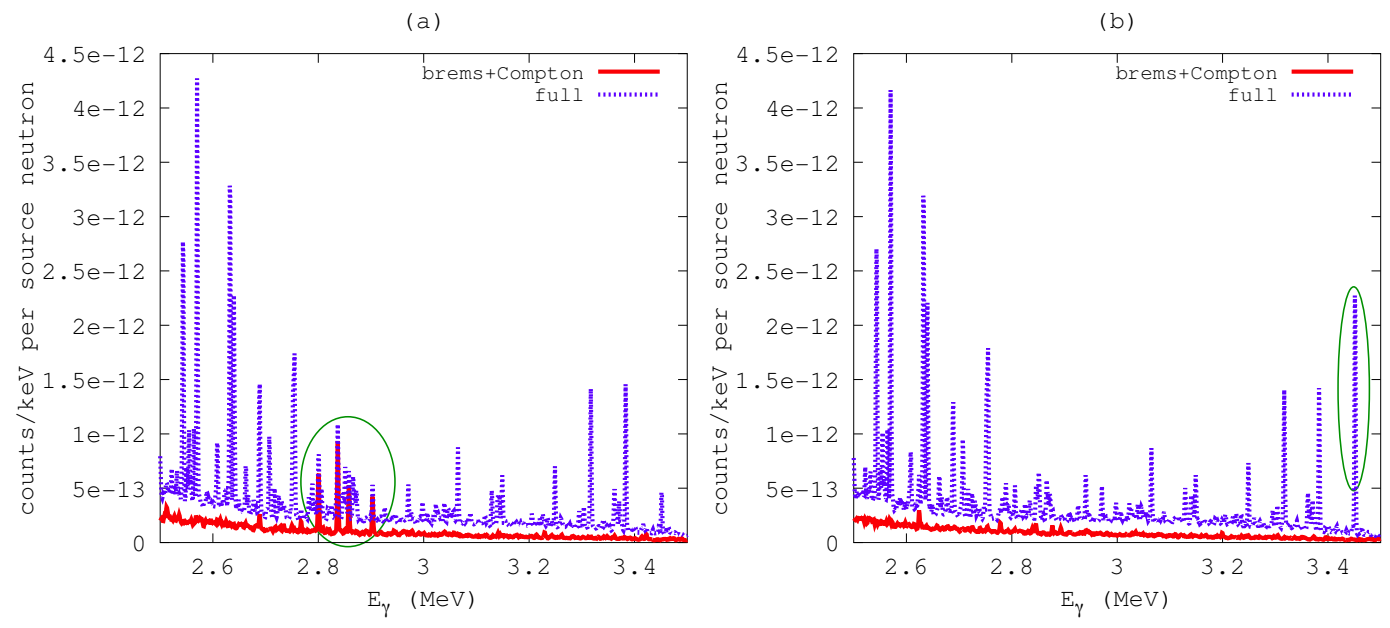

Figure 2: Analyzed spectra obtained from surface source tape augmented with particle tags showing artifacts introduced by high-energy scattered photons in a simulation. The blue curves are the full simulated spectra while the red curves are the bremsstrahlung and Compton scattered portion of the spectra. (a) $\mathrm{NOCOH=1}$ but no automatic weight control; (b) $\mathrm{NOCOH}=0$. 
sure that these Compton events are not undersampled. 55 For this reason, we introduced automatic weight control in MMAPDNG to minimize the variance introduced by such high-energy forward scattered pseudo particles from next event estimators. The particle tag added in the augmented surface source tape can inform a user if any of such artifacts still exists in the simulation results after automatic weight control at each interaction point.

\subsection{Usage and Examples}

MMAPDNG was developed with usability in mind. Towards this goal the new input cards were minimized. There is no specific option for turning energy biasing on or off; instead the BIASENERGY constant is set in the header file as further discussed in Appendix A. The angular biasing is activated by enabling the exponential transform feature in the original MCNPX code by selecting a small stretching parameter as explained in Section 2.2.1.

To turn on the delayed gamma-ray capability from MMAPDNG, a user only needs to pick a thresh value that is greater than one in the activation card ACT. Currently, the code is written to model only delayed gammas from fission products. The gamma rays from the decay of activation products and spontaneous photons are not supported thus the nonfiss parameter should be set to none and the dg parameter should be set to lines. The integer

\footnotetext{
${ }^{5}$ The number of distorted Compton next event pseudo particles depends on both how strongly forward peaking the angular distributions of Compton scattering are and how much the particle weight distributions of the delayed gammas spread. If the weight distribution for gammas at certain energy is broadly spread (due to variance reduction techniques used in the simulation), the probability of getting a distorted Compton next event pseudo particle becomes higher.
} 
part of the thresh value represents the number of fission product pairs to be split as a mean to improve the statistical variances. The code further splits the high-energy delayed gammas (i.e. $E_{\gamma}>2.7 \mathrm{MeV}$ ) from fission products by the inverse of the fractional part of the thresh value round to closest integer. Notice that this is not the automatic weight control mentioned in Section 2.2.3 in which splitting is based on the energy of the emitted delayed gamma of the fission product that is undergoing radioactive decay.

Listing 1: Example of ACT card that activates MMAPDNG code

act nonfiss=none dn=library dg=lines thresh=2.25

Listing 1 shows an example in which each high-energy delayed gamma is split into four (i.e. $1 / 0.25=4$ ) tracks of equally divided weighted probability and each fission is sampled twice (i.e. 2 in the integer part of thresh) with equally divided weighted probability to give four parent fission products which subsequently decay into other fission products.

The remaining user input parameters related to MMAPDNG are the new keywords introduced to the surface source write (i.e. SSW) and surface source read (i.e. SSR) cards. The particle tag is always allocated in the augmented surface source tape but the reaction tag is only activated when the simulation has one or more tallies with special treatment card (i.e. FT card) that enables tagging (e.g. tag 3). The new keywords for the SSW card are wtm, don and ncy. These optional keywords for SSW can be used to select a generation time window for delayed gammas.

The example shown in Listing 2 illustrates the usage of these new keywords. An arbitrary tally (i.e. f101) has a special treatment card to turn on the particle tag feature and a surface source write SSW card is set to discard 
delayed gammas generated during 10.1 seconds dead time (i.e. don=101+7) at the beginning of each 20 -second cycle (i.e. wtm=2+9) for 15 minutes (i.e. $\mathrm{ncy}=900+8$ ). Any delayed gammas generated after the time defined by ncy are discarded.

Listing 2: Example of using new keywords in SSW card

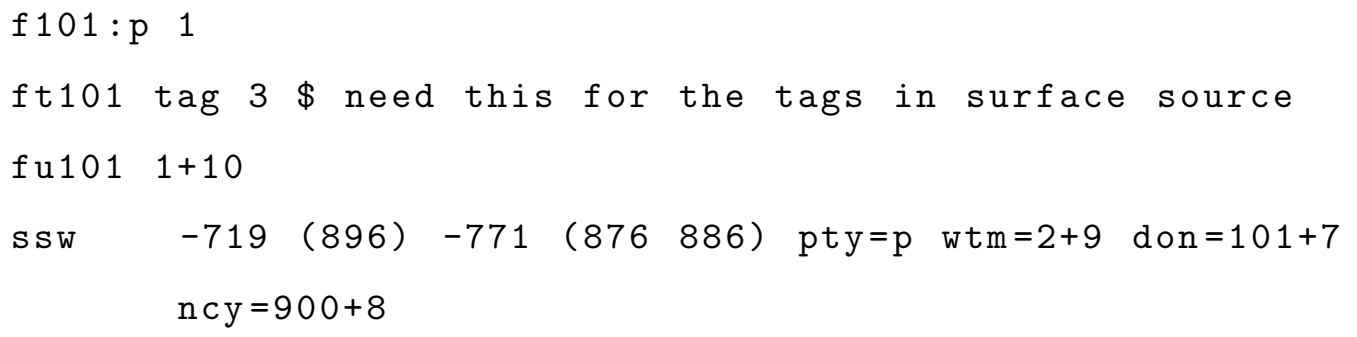

While these keywords for SSW card are optional, they are applicable in most simulations of delayed gamma-ray emission. In fact, when one is interested only in delayed gamma rays, one can turn off the prompt gamma production by setting PWT value to $-1+6$, which additionally saves memory space by expunging the neutron induced gamma production cross sections from the cross section data.

The augmented surface source tape generated on a detector surface can be used in many ways. It can be (a) fed directly into another MCNPX run to simulate a measured spectrum; (b) read by the external code mapsrc to produce an unfolded spectrum which can further be folded into a measured spectrum by another external program such as detResp; (c) read by mapsrc to generate source definition input cards for detector response matrix calculation; and, (d) read by mapsrc to perform other statistical analyses. The only new keyword for the SSR card is dng, which is mandatory and discussed in Section 2.2.3. The external analysis codes mapsrc and detResp are discussed 
in Appendix B as they are not part of the MMAPDNG code integrated into MCNPX.

\section{Results}

In this section, we present and discuss the results of benchmarking the MMAPDNG code to CINDER90 and experimental delayed gamma rays measurements. The MMAPDNG code was extensively used for simulating delayed gamma-ray measurements on used nuclear fuel assemblies and the results of that study will be published elsewhere.

\subsection{Benchmarking Against CINDER90}

As the original delayed gamma code in MCNPX fails to produce satisfactory results due to implementation flaws and other possible bugs mentioned in Section 1.1, we did not compare the new MMAPDNG results against the original delayed gamma code in MCNPX. Instead, we only compared the computing speed and memory usage with the original code and benchmarked the results of the new MMAPDNG code against CINDER90 calculations and experimental data. For the comparisons a simple model consist-

ing of a very narrow, long and extremely low density ${ }^{235} \mathrm{U}$ cyclinder with a mono-directional, mono-energetic thermal neutron source emitting along the centerline of the cylinder was modeled. Calculated were the thermal fission rate and the delayed gamma-ray spectrum emitted from this cylinder.

Table 1 lists the thresh values used in the simulations as well as the number of source particles simulated (i.e. nps) in a $\sim 10$-minute run (i.e. ctm $\approx 10$ ). The thresh value represents the fraction of discrete delayed gamma lines retained by the original delayed gamma code in MCNPX when its value is less 
Table 1: Comparison of sampling speed of fission induced delayed gamma rays in MCNPX version 2.7.0 using the original code versus the new MMAPDNG module. 
duced from GBytes to 7 8 MBytes depending on whether ENDF/B-VI or ENDF/B-VII.1 decay data are used.

To validate the delayed gamma-ray results produced by the MMAPDNG code, we ran a CINDER90 calculation for the same problem and obtained the fission product yields (for the time when the neutron source was off). We then benchmarked the MMAPDNG result against the corresponding CINDER90 result by converting the MMAPDNG computed intensity of a gamma-ray line from a fission product back into its accumulated fission product yield after normalizing the results by the number of induced fissions. Table 2 compares the accumulated fission product yields for eleven isotopes for two different irradiation/counting schemes: (a) 15 minutes (source) on, 30 minutes off (counting), repeated 5 times, and (b) 10 seconds on, 10 seconds off, repeated 30 times.

The results from MMAPDNG and CINDER90 agree within 3\% (for most isotopes within 1\%) except for the shortest half-lived fission products on the list (i.e. ${ }^{97} \mathrm{Y}$ in the 15 -min/30-min on/off irradiation and ${ }^{86} \mathrm{Br}$ in the 10 -sec/10-sec on/off irradiation). The discrepancy for ${ }^{97} \mathrm{Y}$ can easily be explained by the fact that the gammas from short half-live fission products have large statistical variances in a measurement with much longer irradiation/counting times while the discrepancy for ${ }^{86} \mathrm{Br}$ can be explained by the different half-lives of its precursor (i.e. ${ }^{86} \mathrm{Se}$ ) in MMAPDNG and CINDER90. The half-life of ${ }^{86} \mathrm{Se}$ in MMAPDNG is 14.3 seconds as reported in ENDF/BVII.1 while it is 15.3 seconds in the cinder dat file from CINDER90. More discrepancies in the decay data from different data libraries are discussed in Section 3.2 . 
Table 2: Comparison of MMAPDNG and CINDER90 results

\begin{tabular}{|c|c|c|c|c|c|c|c|}
\hline \multirow[b]{2}{*}{ Isotope } & \multirow[b]{2}{*}{$\begin{array}{c}\mathrm{T}_{1 / 2} \\
\text { (minutes) }\end{array}$} & \multicolumn{3}{|c|}{$\begin{array}{l}15 \text { minutes on, } 30 \text { minutes off, } \\
3.75 \text { hours }\end{array}$} & \multicolumn{3}{|c|}{$\begin{array}{l}10 \text { seconds on, } 10 \text { seconds off, } \\
10 \text { minutes }\end{array}$} \\
\hline & & CINDER90 & MMAPDNG & $\%$ diff & CINDER90 & MMAPDNG & $\%$ diff \\
\hline${ }^{86} \mathrm{Br}$ & 0.92 & $2.01 \times 10^{-3}$ & $1.99 \times 10^{-3}$ & -1.10 & $8.19 \times 10^{-3}$ & $8.49 \times 10^{-3}$ & 3.66 \\
\hline${ }^{89} \mathrm{Kr}$ & 3.15 & $1.32 \times 10^{-2}$ & $1.32 \times 10^{-2}$ & 0.03 & $1.37 \times 10^{-2}$ & $1.39 \times 10^{-2}$ & 0.94 \\
\hline${ }^{90} \mathrm{Rb}$ & 2.63 & $1.33 \times 10^{-2}$ & $1.33 \times 10^{-2}$ & 0.10 & $1.32 \times 10^{-2}$ & $1.32 \times 10^{-2}$ & 0.11 \\
\hline${ }^{90 \mathrm{~m}_{\mathrm{Rb}}}$ & 4.30 & $5.09 \times 10^{-3}$ & $5.10 \times 10^{-3}$ & 0.03 & $3.16 \times 10^{-3}$ & $3.19 \times 10^{-3}$ & 1.06 \\
\hline${ }^{91} \mathrm{Rb}$ & 0.97 & $5.67 \times 10^{-3}$ & $5.68 \times 10^{-3}$ & 0.04 & $2.42 \times 10^{-2}$ & $2.44 \times 10^{-2}$ & 0.96 \\
\hline${ }^{93} \mathrm{Rb}$ & 0.10 & $3.32 \times 10^{-4}$ & $3.42 \times 10^{-4}$ & 2.95 & $1.62 \times 10^{-2}$ & $1.63 \times 10^{-2}$ & 0.84 \\
\hline${ }^{95} \mathrm{Sr}$ & 0.40 & $2.02 \times 10^{-3}$ & $2.01 \times 10^{-3}$ & -0.48 & $2.51 \times 10^{-2}$ & $2.51 \times 10^{-2}$ & 0.14 \\
\hline${ }^{95} \mathrm{Y}$ & 10.30 & $3.73 \times 10^{-2}$ & $3.75 \times 10^{-2}$ & 0.55 & $8.22 \times 10^{-3}$ & $8.33 \times 10^{-3}$ & 1.36 \\
\hline${ }^{97} \mathrm{Y}$ & 0.06 & $1.84 \times 10^{-4}$ & $1.92 \times 10^{-4}$ & 4.50 & $1.27 \times 10^{-2}$ & $1.27 \times 10^{-2}$ & -0.06 \\
\hline${ }^{106} \mathrm{Tc}$ & 0.59 & $2.81 \times 10^{-4}$ & $2.83 \times 10^{-4}$ & 0.49 & $1.85 \times 10^{-3}$ & $1.89 \times 10^{-3}$ & 2.44 \\
\hline${ }^{136} \mathrm{Te}$ & 0.29 & $3.85 \times 10^{-4}$ & $3.80 \times 10^{-4}$ & -1.35 & $6.55 \times 10^{-3}$ & $6.61 \times 10^{-3}$ & 0.91 \\
\hline
\end{tabular}


Table 3: Fission Product Yields of ${ }^{86} \mathrm{Br}$ and ${ }^{97} \mathrm{Y}$ for ${ }^{235} \mathrm{U}$ Thermal Fission

\begin{tabular}{l|c|l|l|l|l|l}
\hline & \multicolumn{3}{|c}{ Independent Fission Product Yield } & \multicolumn{3}{c}{ Cumulative Fission Product Yield } \\
\hline $\mathrm{FP}$ & JENDL-4.0 & JEFF-3.1.1 & ENDF/B-VII.1 & JENDL-4.0 & JEFF-3.1.1 & ENDF/B-VII.1 \\
\hline${ }^{86} \mathrm{Br}$ & $4.588 \times 10^{-3}$ & $6.179 \times 10^{-3}$ & $2.297 \times 10^{-3}$ & $1.815 \times 10^{-2}$ & $1.875 \times 10^{-2}$ & $1.595 \times 10^{-2}$ \\
${ }^{97} \mathrm{Y}$ & $5.895 \times 10^{-3}$ & $6.188 \times 10^{-3}$ & $1.570 \times 10^{-2}$ & $2.354 \times 10^{-2}$ & $2.080 \times 10^{-2}$ & $4.890 \times 10^{-2}$ \\
\hline
\end{tabular}

\subsection{Comparison to Experimental Data and Selection of Nuclear Data Li- brary}

Further examination of the nuclear decay data and fission product yield data for ${ }^{86} \mathrm{Br}$ and ${ }^{97} \mathrm{Y}$ revealed discrepancies between different data libraries. For instance, the independent fission product yield of ${ }^{86} \mathrm{Br}$ for thermal fission of ${ }^{235} \mathrm{U}$ in JEFF-3.1.1 is $\sim 1.7$ times higher than that in ENDF/B-VII.1 while the independent fission product yield for ${ }^{97} \mathrm{Y}$ in JENDL-4.0 is $\sim 62 \%$ less than that in ENDF/B-VII.1 as seen in Table 3. One should also note that the independent and cumulative fission product yields of ${ }^{86} \mathrm{Br}$ for thermal fission of ${ }^{235} \mathrm{U}$ in cinder. dat of CINDER90 are both significantly different from the values in the above data libraries (See Table 3).

It is not surprising that discrepancies between different decay and fission product yield data libraries exist for many short-lived fission products because the measurements for these fission products are rather difficult. In order to further test our MMAPDNG code, we modeled delayed gamma measurements that had been carried out at the Idaho Accelerator Center using a photoneutron source. Figure 3 shows measured and simulated delayed gamma-ray spectra emitted from a depleted uranium plate after irradiation with thermalized neutrons.

Figure 3(a) shows that there are several discrepancies between the simulated and measured spectra. Most of the high-energy peaks from the decay of 


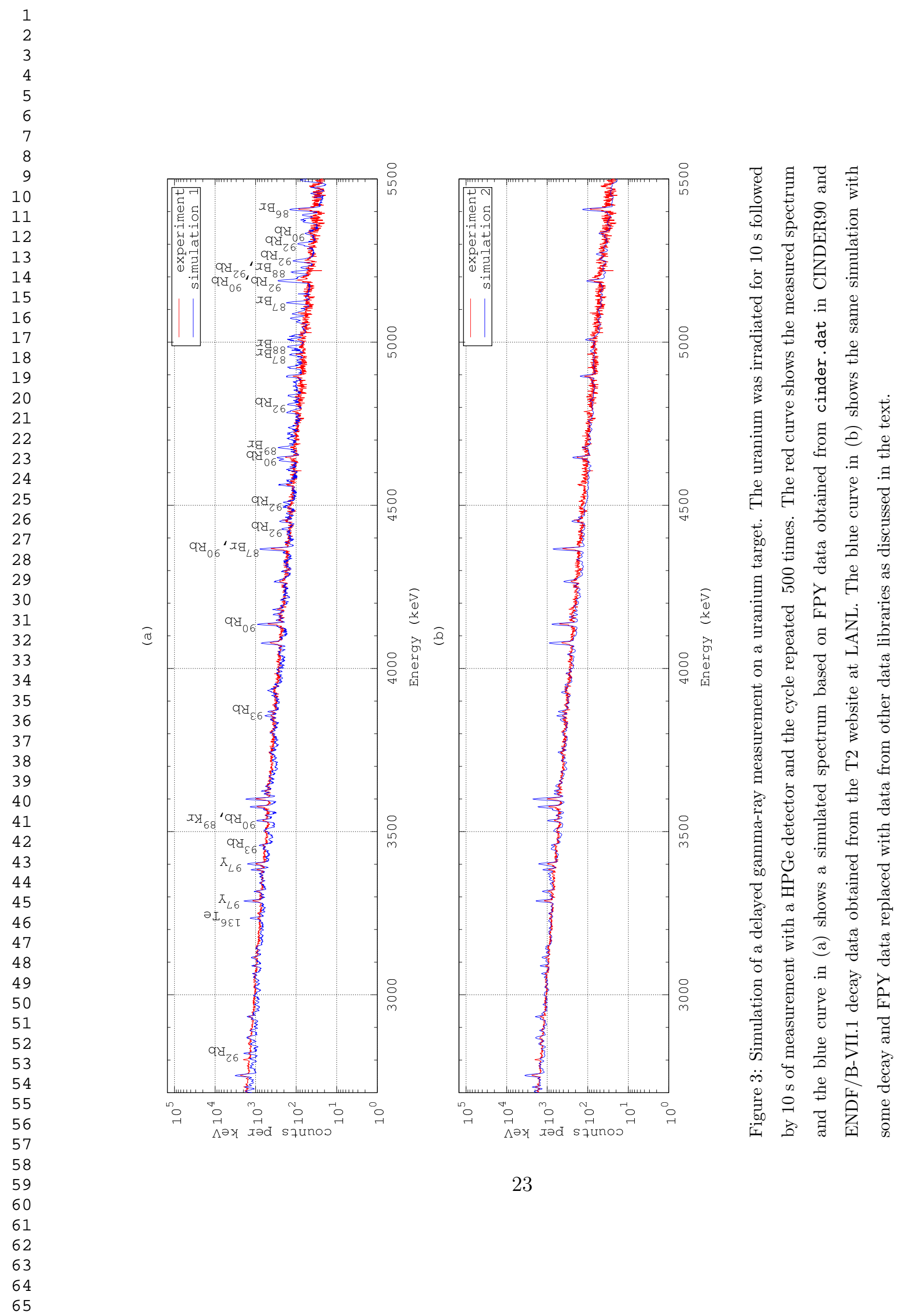


${ }^{136} \mathrm{Te},{ }^{87} \mathrm{Br},{ }^{88} \mathrm{Br},{ }^{89} \mathrm{Br}$ and ${ }^{92} \mathrm{Rb}$ are not observed in the experimental spectrum. In addition, the simulated ${ }^{97} \mathrm{Y}$ and ${ }^{86} \mathrm{Br}$ peaks are more prominent than their measured peaks. The gamma intensities of these fission products from both ENDF-B/VI.8 and ENDF-B/VII.1 decay data are comparable. This suggests that there are discrepancies between the ${ }^{97} \mathrm{Y}$ and ${ }^{86} \mathrm{Br}$ FPY data in cinder. dat and their true values. Furthermore, the simulated ${ }^{90} \mathrm{Rb}$ and ${ }^{93} \mathrm{Rb}$ peaks are also more prominent than their measured peaks. This may be due to wrong gamma intensities for delayed gammas from ${ }^{93} \mathrm{Rb}$ at $3.458 \mathrm{MeV}$ and $3.868 \mathrm{MeV}$ in the ENDF-B/VI.8 data library.

To illustrate the significance of these discrepancies in nuclear data discovered in our benchmarking efforts, Figure 3(b) shows the simulated spectrum with the following changes in the simulation: (i) the line data of ${ }^{136} \mathrm{Te},{ }^{87} \mathrm{Br}$, ${ }^{88} \mathrm{Br},{ }^{89} \mathrm{Br}$ and ${ }^{92} \mathrm{Rb}$ are replaced with the 25 -group gamma yield data from cinder.dat; (ii) the ${ }^{97} \mathrm{Y},{ }^{97 \mathrm{~m}} \mathrm{Y}$ and ${ }^{97} \mathrm{Sr}$ FPY data in cinder.dat are replaced with data from JEFF-3.1.1; and, (iii) the ${ }^{86} \mathrm{Br},{ }^{86 \mathrm{~m}} \mathrm{Br}$ and ${ }^{86} \mathrm{As}$ FPY data for ${ }^{235} \mathrm{U}$ thermal fission and ${ }^{239} \mathrm{Pu}$ high-energy fission in cinder . dat are replaced with data from ENDF-B/VII.1; (iv) the gamma intensities data for ${ }^{93} \mathrm{Rb}$ decay from ENDF-B/VI.8 are replaced with data from ENDF-B/VII.1.

Although the simulated spectrum in Figure 3(b) is a better match of the measured spectrum, we should note that the uncertainties in nuclear data are simply large and, as discussed above, data were selected from the libraries to give the best agreement of simulated and measured spectrum. Nevertheless, we show that MMAPDNG can reproduce the measured spectrum and that the accuracy of nuclear data is crucial for obtaining the correct simulated spectrum. 


\section{Conclusions}

A new code, MMAPDNG (Memory-Mapped Delayed Neutron and Gamma code), has been developed that is fully integrated into the radiation transport code MCNPX and replaces the existing routine for calculating delayed gamma-ray emission following nuclear fission. MCNPX with MMAPDNG allows the simulation of delayed gamma measurements and of complex problems such as the modeling of high-energy, delayed gamma-ray spectroscopy for the assay of nuclear materials. Its approach is different from existing codes. The fission product yield data are converted to cumulative distribution functions while the delayed emission data are saved as pre-sorted and pre-calculated data from which the delayed gamma-ray emission times and energies are sampled. The MMAPDNG code employs automatic energy and angular biasing and is orders of magnitude faster than the original code yet consumes orders of magnitude less memory. An important feature of the code is the use of a surface source tape with particle tag that provides extra information for analyzing simulation results and to identify artifacts in the spectra. The MMAPDNG code has been benchmarked against CINDER90 and good agreement has been found. In addition, an experimental delayed gamma measurement has been simulated. Uncertainties in the nuclear data libraries and differences between them are reflected in the differences between simulated and experimental data.

We have submitted the source code of MMAPDNG to the MCNPX development team as we wish to make our code available to other researchers. Currently, the submitted MMAPDNG code can generate only delayed gammas but the code is extensible to other delayed particles such as neutron and 
electrons. To make the code most easily accessible, it should be ported to MCNP6. For completeness, delayed gamma emissions from neutron activation of non-fissionable materials and spontaneous fission of actinides should be implemented too. It would be beneficial to continue the development of these extensions based on our existing MMAPDNG framework.

\section{Appendix A. Code to create the file-backed memory-mapped de- layed gamma-ray database: dng}

The dng code is an external program used to create cinder.mmap, the memory-mapped file for the decay and FPY data. cinder.mmap is stored under \$TMPDIR which is usually /tmp. The dng code must run as least once to generate the required cinder.mmap file for MMAPDNG to work on a system. Every slave MPI node must have a copy of cinder . mmap in its \$TMPDIR directory. The dng code is thus distributed as part of the MMAPDNG package.

dng uses the DATAPATH and ENDFBVII environments to look up the following files: cinder.dat and cindergl.dat. If only DATAPATH is defined in the environment, dng will look for both files under \$DATAPATH. If ENDFBVII is also defined, dng will look for cindergl.dat under \$ENDFBVII instead. When run with an option "-c", dng creates \$TMPDIR/cinder.mmap. When run without any option, dng attempts to open \$TMPDIR/cinder.mmap and check if it can map the file.

As mentioned in Section 2.2.1, a user can change the BIASENERGY constant in cidDat.h files for dng and MCNPX. After changing the header files under dng directory and MCNPX source directory, the dng and MCNPX 
executables must be recompiled and the cinder.mmap must be re-created before running a simulation.

\begin{abstract}
Appendix B. Auxiliary data analysis programs that are not part of MMAPDNG: mapsrc and detResp

mapsrc is an external program used to analyze the data in the augmented surface source tape file while detResp is an external program used to generate a simulated spectrum from a gamma ray detector if a detector response matrix and the parameters for its Gaussian energy broadening function are provided. The usage of mapsrc or detResp can simply be obtained by running the command without any option.
\end{abstract}

\title{
Acknowledgements
}

The authors would like to thank Prof. Alan Hunt and Edward Reedy (both Idaho State University) for providing the experimental delayed gammaray spectra for benchmarking the code. This work was supported by the Next Generation Safeguards Initiative, Office of Nonproliferation and International Security, National Nuclear Security Administration and performed under the auspices of the U. S. Department of Energy by the Lawrence Berkeley National Laboratory under Contract No. DE-AC02-05CH11231.

\section{References}

[1] D. Beddingfield, F. Cecil, Identification of fissile materials from fission product gamma-ray spectra, Nuclear Instruments and Meth- 
ods in Physics Research Section A: Accelerators, Spectrometers, Detectors and Associated Equipment 417 (2-3) (1998) 405 - 412. doi:http://dx.doi.org/10.1016/S0168-9002(98)00781-5. URL http://www.sciencedirect.com/science/article/pii/ S0168900298007815

[2] E. B. Norman, S. G. Prussin, R.-M. Larimer, H. Shugart, E. Browne, A. R. Smith, R. J. McDonald, H. Nitsche, P. Gupta, M. I. Frank, T. B. Gosnell, Signatures of fissile materials: high-energy $\gamma$ rays following fission, Nuclear Instruments and Methods in Physics Research Section A: Accelerators, Spectrometers, Detectors and Associated Equipment 521 (2-3) (2004) 608 - 610. doi:http://dx.doi.org/10.1016/j.nima.2003.10.097. URL http://www.sciencedirect.com/science/article/pii/ S0168900203028948

[3] D. Slaughter, M. Accatino, A. Bernstein, J. Church, M. Descalle, T. Gosnell, J. Hall, A. Loshak, D. Manatt, G. Mauger, T. Moore, E. Norman, B. Pohl, J. Pruet, D. Petersen, R. Walling, D. Weirup, S. Prussin, M. McDowell, Preliminary results utilizing high-energy fission product $\gamma$-rays to detect fissionable material in cargo, Nuclear Instruments and Methods in Physics Research Section B: Beam Interactions with Materials and Atoms 241 (1-4) (2005) 777 - 781. doi:http://dx.doi.org/10.1016/j.nimb.2005.07.236. URL http://www.sciencedirect.com/science/article/pii/ S0168583X05013212 
[4] L. W. Campbell, L. E. Smith, A. C. Misner, High-energy delayed gamma spectroscopy for spent nuclear fuel assay, IEEE Transactions in Nuclear Science 58 (2011) 231.

[5] G. McKinney, J. Durkee, J. Hendricks, M. James, R. Johns, D. B. Pelowitz, L. S. Waters, MCNPX 2.6.x features (2006-2007), Tech. Rep. LA-UR-07-2053, LANL (April 2007). URL https://mcnpx.lanl.gov/opendocs/misc/LA-UR-07-2053.pdf

[6] M. Chin, N. Spyrou. Black-box monte carlo: Implications on simulation of neutron reactions [online] (October 2007) [cited Oct 29, 2013].

[7] W. B. Wilson, S. T. Cowell, T. R. England, A. C. Hayes, P. Moller, A manual for CINDER'90 Version 07.4 codes and data, Tech. Rep. LAUR-07-8412, LANL (March 2008).

[8] V. Mozin, Delayed gamma-ray assay for nuclear safeguards, Ph.D. thesis, University of California, Berkeley (2011).

[9] V. Mozin, J. Vujic, A. Hunt, S. Tobin, Development and validation of the discrete prompt and delayed gamma-ray source term and detector response calculation technique, in: International Conference on Mathematics and Computational Methods Applied to Nuclear Science and Engineering (MC 2011), 2011.

[10] V. Mozin, L. Campbell, A. Hunt, B. Ludewigt, Delayed gamma-ray spectroscopy for spent nuclear fuel assay, Journal of Nuclear Materials Management 40 (3) (2012) 78. 
[11] D. B. Pelowitz (Ed.), MCNPX ${ }^{\mathrm{TM}}$ User's Manual Version 2.7.0, no. LACP-11-00438, Los Alamos National Laboratory, 2011.

[12] D. B. Pelowitz, J. W. Durkee, J. S. Elson, M. L. Fensin, J. S. Hendricks, M. R. James, R. C. Johns, G. W. McKinney, S. G. Mashnik, J. M. Verbeke, L. S. Waters, T. A. Wilcox, MCNPX ${ }^{\mathrm{TM}} 2.7 .0$ extensions, Tech. Rep. LA-UR-11-02295, Los Alamos National Laboratory (April 2011).

[13] X-5 Monte Carlo Team, MCNP - A General Monte Carlo N-Particle Transport Code, Version 5 Volume I: Overview and Theory, Los Alamos National Laboratory (April 2003). 